\title{
Vendor Management Plan
}

National Cancer Institute

\section{Source}

National Cancer Institute. Vendor Management Plan. NCI Thesaurus. Code C126094.

A plan to document overall management strategy for vendors used to conduct clinical trial-related activities. 